\title{
Pediatric non-rhabdomyosarcoma soft tissue sarcomas
}

\author{
Brandon R. Mancini • Kenneth B. Roberts
}

Received: 26 October 2012 / Accepted: 9 November 2012 /Published online: 14 December 2012

(C) Springer-Verlag Berlin Heidelberg 2012

Keywords Pediatric · Soft tissue sarcoma ·

Non-rhabdomyosarcoma $\cdot$ Radiotherapy

\section{Background}

Soft tissue sarcomas encompass a diverse group of mesenchymal tumors, some of which have unique biology and epidemiology within the pediatric age group. Rhabdomyosarcomas are the best characterized and studied with relatively good sensitivity to both chemotherapy and radiotherapy. To distinguish the other broad category of pediatric tumors derived from connective tissue, the cumbersome term, nonrhabdomyosarcoma soft tissue sarcomas (NRSTS), has been coined. Many, but not all NRSTS, share characteristics with adult-type soft tissue sarcomas (STS) leading to a wealth of treatment principles that cross the age spectrum. But because of developmental toxicities especially from radiotherapy, there are considerable modifications in treatment philosophy with the management of NRSTS. Unlike rhabdomyosarcoma, where well conducted randomized trials have progressively refined the dose, fractionation, and volume of radiotherapy in a risk adapted manner, the evidence for optimal management of NRSTS is still evolving. Relative to the adult management of STS, the oncologist needs to be more mindful of the late

B. R. Mancini • K. B. Roberts

Department of Therapeutic Radiology,

Yale University School of Medicine, New Haven, CT, USA

B. R. Mancini

e-mail: brandon.mancini@yale.edu

K. B. Roberts $(\bowtie)$

Smilow Cancer Hospital LL, 15 Park Street,

New Haven, CT 06510, USA

e-mail: kenneth.roberts@yale.edu effects of treatment, which include concerns for normal growth, fertility, and risk for secondary malignancy. But at what age should STS be treated more aggressively with radiotherapy is difficult to know as NRSTS clinical trials extend eligibility to young adults. This review will provide an overview of evolving knowledge regarding NRSTS while focusing on the radiotherapeutic aspects.

\section{Epidemiology}

Soft tissue sarcomas represent $6-7 \%$ of all pediatric cancers (defined as those occurring before age 20 years). Of these, $40 \%$ are rhabdomyosarcoma and $60 \%$ are NRSTS [1-3]. The USA's Surveillance, Epidemiology, and End Results (SEER) program provides some of the most comprehensive cancer epidemiologic data available [4]. Of the European Tumor Registries, only data published from Germany have sufficient detail to distinguish rhabdomyosarcoma from NRSTS [5]. Overall, NRSTS comprise approximately 3$3.5 \%$ of all pediatric cancers with 550 to 600 new cases diagnosed each year in the USA. The incidence rate over time has remained steady in the range of six to eight cases per million population. In Germany, the incidence rate is slightly less at five cases per million persons. Data from SEER based on the time period 1975-1995 suggest that NRSTS has had a propensity to dominate in older pediatric age groups (10-14 years and 15-19 years, specifically) as part of the incidence curves for STS leading into adulthood. In fact, clinical features for soft tissue sarcomas are similar between children and adults, except that survival rates diminish for patients over age 50 years [6]. See Fig. 1, depicting the distribution of STS subtypes, location of disease, and staging by 10 -year age intervals.

The survival of patients with NRSTS is dependent on the histologic subtype to a certain degree. Figure 2 depicts the 
Fig. 1 a Distribution of histologic subtypes by 10 -year age groups. b Distribution of primary tumor sites by 10 -year age groups. c Distribution of tumor stage by 10 -year age groups. Rhabdomyosarcomas (RMS); fibroblastic and myofibroblastic tumors (fibroblastic); fibrohistiocytic tumors (fibrohistiocytic); malignant peripheral nerve sheath tumors (NST); Kaposi sarcoma (Kaposi); Ewing family tumors ( $p P N E T)$; extraneral rhabdoid tumor (rhabdoid); liposarcomas (liposarcoma); synovial sarcomas (synovial); blood vessel tumors (blood vessel); alveolar soft parts sarcoma

(ASPS); miscellaneous/unspecified soft tissue sarcomas including other fibromatous neoplasms (miscellaneous). From ref. [6].

This material is reproduced with permission of John Wiley \&

Sons, Inc.
A

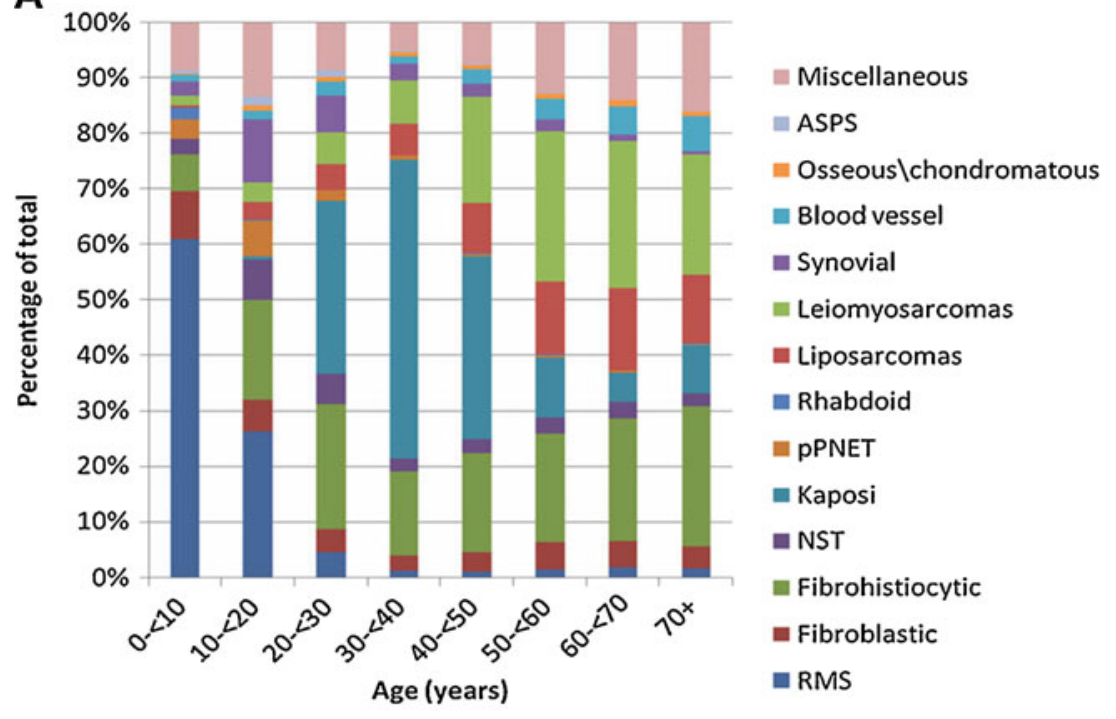

B

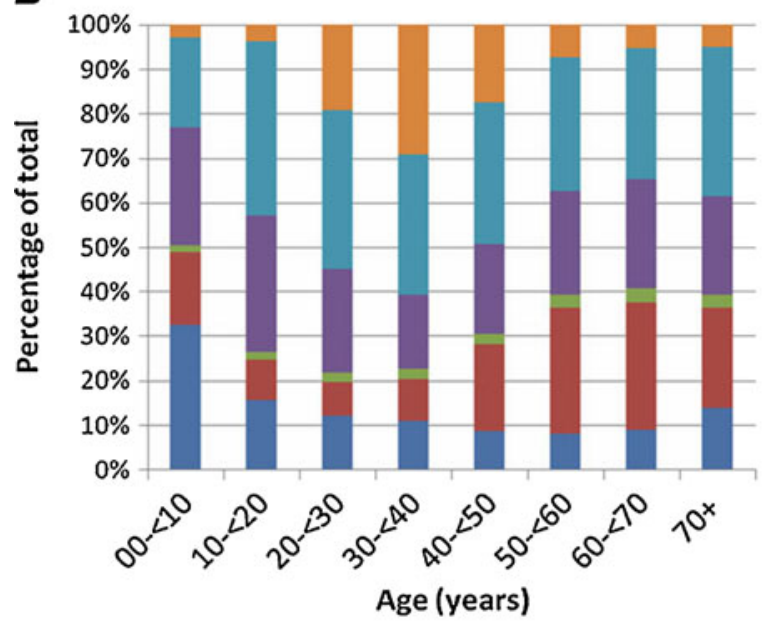

= others

Extremities

- Trunk

= intra_thoracic

aintra_abdominal

- Head_neck

C

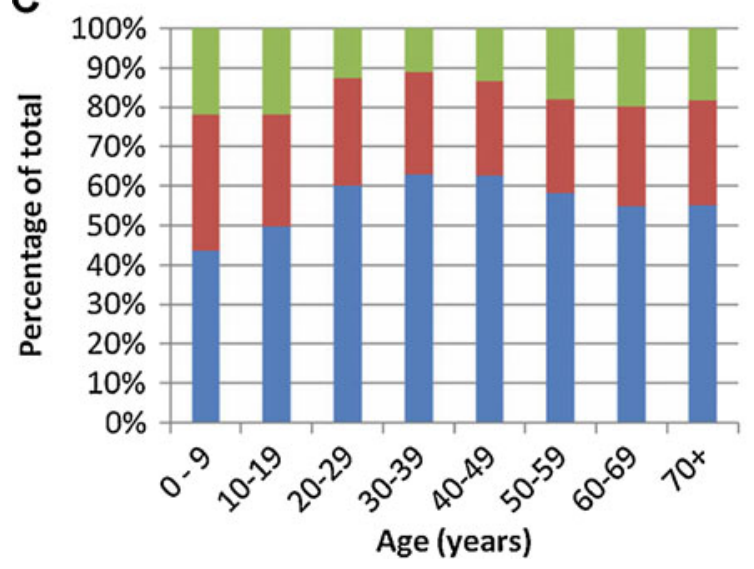

Distant

Regional

- Localized 5-year survival rates by histology across the age spectrum. Within the pediatric age group, patients with fibrosarcomas, synovial cell sarcomas tend to have better survivals than malignant peripheral nerve sheath tumors and extra-osseous peripheral neuroectodermal tumors. Although the trends in survival improvements within the SEER database are hard to decipher as to differences between rhabdomyosarcoma and NRSTS, pediatric soft tissue sarcomas have been associated with improving survival over time. Specifically, for patients younger than 16 years of age who were diagnosed 


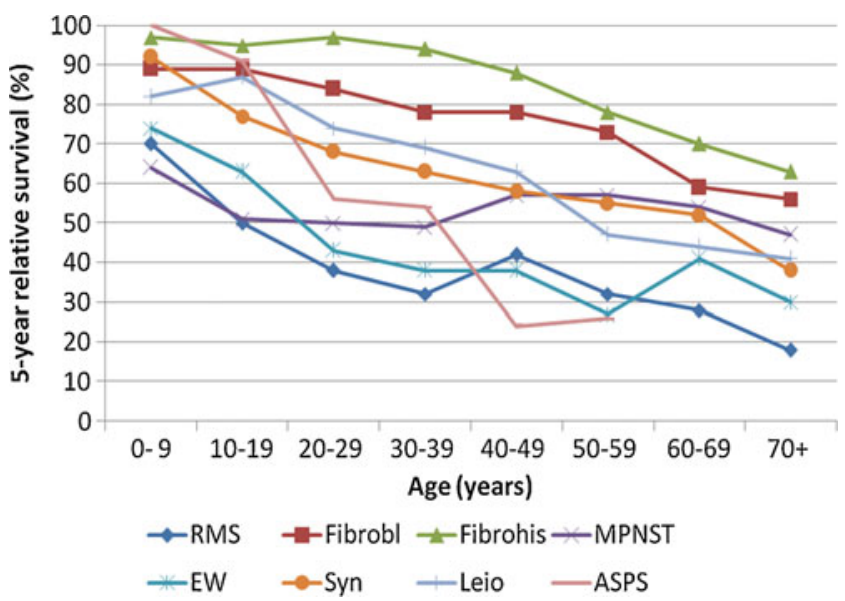

Fig. 2 Five-year survival rates for various soft tissue sarcomas across the age spectrum. From ref. [6]. This material is reproduced with permission of John Wiley \& Sons, Inc.

with a soft tissue sarcoma in 1975-1977, the adjusted 5-year survival was $61 \%$. For those diagnosed between 1996 and 2004 , there was a statistically significant improvement in the 5-year survival to $74 \%[7,8]$. But, in a SEER comparison of the time periods 1975 to 1984 with 1985 to 1994 , there was no improvement in survival rates for NRSTS [4]. In addition, there have been relatively few patients with NRSTS who have been enrolled in cooperative group clinical trials. While the Children's Oncology Group (COG) ARST0332 trial closed in February 2012 with 588 patients enrolled over 5 years, prior to that time less than 200 patients had been enrolled in only three prospective clinical trials in the USA $[9,10]$. ARST0332 was a comprehensive study of NRSTS in the USA across different stages and risk strata, representing current treatment concepts, and will be discussed further below.

Given the common clinical features of many pediatric and adult STS, it is important to note that these sarcomas are overall rare with approximately 9,000-9,500 cases diagnosed in the USA annually (less than $1 \%$ of malignant tumors). In those diagnosed with an STS across the age spectrum, approximately one third die from the disease. Sites of sarcoma development are distributed in the following way: $46 \%$ lower extremity, $19 \%$ torso, $14 \%$ upper extremity, $13 \%$ retroperitoneum, and $8 \%$ head and neck. In general, sarcomas tend to be locally infiltrative and nodal metastases are uncommon, especially for small, low-grade tumors. There is some tendency for regional nodal metastases in the synovial cell and epithelioid histological subtypes (as there is for rhabdomyosarcomas). Overall, the most common site for distant metastatic disease is the lung. In comparing pediatric NRSTS and adult STS, the most common histologic subtype for both groups is synovial sarcoma. Similarly to adult sarcomas, pediatric NRSTS tend to occur in the extremities or trunk and present as a mass or other symptoms due to invasion of an adjacent structure.

\section{Pathology}

Many of the pediatric NRSTSs have characteristic cell types. The World Health Organization classification utilizes lines of differentiation in order to categorize NRSTS tumors into adipocytic, fibroblastic/myofibroblastic, fibrohistiocytic, smooth muscle, pericytic (perivascular), and vascular types, as well as tumors of uncertain differentiation [11]. NRSTSs often are difficult for pathologists to classify, and there is wide intra-observer variation [12]. Furthermore, a reasonable number of NRSTSs display no cellular differentiation and care therefore referred to as undifferentiated sarcomas or sarcomas not otherwise specified. Of the poorly differentiated STSs, pleomorphic sarcoma is now the preferred term for malignant fibrous histiocytoma, which tends to be more common in adults than in children. Immunohistochemistry and molecular genetics are often utilized to better characterize soft tissue sarcomas.

For most children with NRSTS, the etiology or cause of tumor development is uncertain. Some cases may be linked with prior radiation exposure, chemical exposure, iatrogenic or disease-causes immunosuppression, and neurofibromatosis, with the latter group having a $2-16 \%$ risk of developing a malignant peripheral nerve sheath tumor (MPNST) in their lifetime. The development of sarcomas in patients with neurofibromatosis has been associated with chromosome 17 deletions [1]. Furthermore, familial Li-Fraumeni syndrome, with deletions affecting the p53 tumor suppressor gene, has been linked to the development of both rhabdomyosarcoma and NRSTS. Finally, gastrointestinal stromal tumors are of particular interest, albeit rare in the pediatric population, as its associated c-kit oncogene mutation has been successfully treated with imatinib, a prototype tyrosine kinase inhibitor [13]. While not yet tested in pediatric NRSTS, there are a variety of other molecular targets and associated agents under investigation in adult soft tissue sarcomas including platelet derived growth factor receptorA (sunitinib), Raf kinase (sorafenib), mTOR (rapamycin), vascular endothelial growth factor (bevacizumab), heat shock proteins, hedgehog, histone deacetylase, and nucleotide excision repair [14]. Table 1 represents a comprehensive list of NRSTS histologies and associated chromosomal aberrations and/or genes involved [14-31].

This expanding knowledge of the molecular pathogenesis of NRSTS is worth highlighting as this will ultimately be a source for future refinements in risk stratification and treatment. As a first example, primitive neuroectodermal tumors, which are both bone and soft tissue tumors, have a characteristic molecular translocation between the EWS and FLII genes on chromosome 22 and chromosome 11, respectively. This translocation, $t$ $(11 ; 22)(\mathrm{q} 24 ; \mathrm{q} 12)$, forms the EWS/FLI1 gene and leads to activation of an aberrant proliferation program, which 
Table 1 Common cytogenetic changes in non-rhabdomyosarcoma soft tissue sarcomas

\begin{tabular}{|c|c|c|}
\hline Histologic type & Characteristic cytogenetic events & Genes involved \\
\hline Alveolar soft-part sarcoma & $\mathrm{t}(\mathrm{X} ; 17)(\mathrm{p} 11 ; \mathrm{q} 25)$ & ASPSCR1-TFE3 (ASPL-TFE3) fusion \\
\hline $\begin{array}{l}\text { Aggressive fibromatosis } \\
\text { (desmoids tumor) }\end{array}$ & Trisomies 8 and 20 Deletion of $5 q$ & $A P C$ inactivation \\
\hline Lipoma (typical) & $12 \mathrm{q} 15$ rearrangement & HMGA2 (HMGIC) rearrangement \\
\hline Well-differentiated liposarcoma & Ring form of chromosome 12 & \\
\hline \multirow[t]{2}{*}{ Myxoid/round-cell liposarcoma } & $\mathrm{t}(12 ; 16)(\mathrm{q} 13 ; \mathrm{p} 11)$ & FUS-DDIT3 (FUS-CHOP) fusion \\
\hline & $\mathrm{t}(12 ; 22)(\mathrm{q} 13 ; \mathrm{q} 12)$ & EWSR1-DDIT3 (EWS-CHOP) fusion \\
\hline Lipoblastoma & Rearrangement of $8 \mathrm{q} 11-13$ & $\begin{array}{l}\text { PLAG1 gene rearrangements: HAS2/ } \\
\text { PLAG1, } \\
\text { COLIA2/PLAG1 }\end{array}$ \\
\hline Pleomorphic liposarcoma & Complex abnormalities & \\
\hline Malignant fibrous histiocytoma & Complex abnormalities & \\
\hline Myxoid malignant fibrous histiocytoma & Ring form of chromosome 12 & \\
\hline Low-grade fibromyxoid sarcoma & $\mathrm{T}(7 ; 16)(\mathrm{q} 34 ; \mathrm{p} 11)$ & $F U S-B B F 2 H 7$ fusion \\
\hline Leiomyoma (uterine) & $\mathrm{t}(12 ; 14)(\mathrm{q} 15 ; \mathrm{q} 24)$ or deletion of $7 \mathrm{q}$ & $H M G A 2$ (HMGIC) rearrangement \\
\hline Leiomyoma (extrauterine) & Deletion of $1 p$ & \\
\hline Leiomyosarcoma & Deletion of $1 p$ Other complex abnormalities & \\
\hline Monophasic synovial sarcoma & $\mathrm{t}(\mathrm{X} ; 18)(\mathrm{p} 11 ; \mathrm{q} 11)$ & $\begin{array}{l}\text { SS18-SSX1 (SYT-SSX1) or SS18-SSX2 } \\
\quad(S Y T-S S X 2) \text { fusion MYCN over-expression }\end{array}$ \\
\hline Biphasic synovial sarcoma & $\mathrm{t}(\mathrm{X} ; 18)(\mathrm{p} 11 ; \mathrm{q} 11)$ & $\begin{array}{l}\text { SS18-SSX1 (SYT-SSX1) fusion MYCN } \\
\text { overexpression }\end{array}$ \\
\hline Benign schwannoma & Deletion of chromosome 22 & NF2 inactivation \\
\hline $\begin{array}{l}\text { Malignant peripheral nerve sheath tumors, } \\
\text { low grade }\end{array}$ & None & \\
\hline $\begin{array}{l}\text { Malignant peripheral nerve sheath tumors, } \\
\text { high grade }\end{array}$ & Complex abnormalities & \\
\hline Primitive neuroectodermal Tumor & $\mathrm{t}(11 ; 22)(\mathrm{q} 24 ; \mathrm{q} 12) \mathrm{t}(21 ; 22)(\mathrm{q} 12 ; \mathrm{q} 12)$ & $\begin{array}{l}\text { EWSR1-FLI1 (EWS-FLI1) fusion } \\
\text { EWSR1-ERG (EWS-ERG) fusion }\end{array}$ \\
\hline Desmoplastic small round-cell tumor & $\mathrm{t}(11 ; 22)(\mathrm{p} 13 ; \mathrm{q} 12)$ & EWSR1-WT1 (EWS-WT1) fusion \\
\hline Dermatofibrosarcoma protuberans & Ring form of chromosomes 17 and $22 \mathrm{t}(17 ; 22)(\mathrm{q} 21 ; \mathrm{q} 13)$ & $C O L 1 A 1-P D G F B$ fusion \\
\hline Endometrial stromal tumor & $\mathrm{t}(7 ; 17)(\mathrm{p} 15 ; \mathrm{q} 21)$ & JAZF1-SUZ12 (JAZF1-JJAZ1) \\
\hline Gastrointestinal stromal tumor & Monosomies 14 and 22 Deletion of $1 p$ & KIT of PDGFRA mutation \\
\hline Fibrosarcoma, infantile & $\mathrm{t}(12 ; 15)(\mathrm{p} 13 ; \mathrm{q} 26)$ trisomy $8,11,17,20$ & ETV6-NTRK3 fusion \\
\hline Extraskeletal myxoid chondrosarcoma & $\mathrm{t}(9 ; 22)(\mathrm{q} 22 ; \mathrm{q} 12) \mathrm{t}(9 ; 17)(\mathrm{q} 22 ; \mathrm{q} 11)$ & $\begin{array}{l}\text { EWSR1-NR4A3 (EWS-NR4A3) fusion } \\
\text { TAF15-NR4A3 (TAF2N-NR4A3) fusion }\end{array}$ \\
\hline Inflammatory myofibroblastic tumor & $2 \mathrm{p} 23$ rearrangement & $\begin{array}{l}\text { ALK fusion to TPM3, TPM4, } \\
\text { clathrin and other genes }\end{array}$ \\
\hline Clear cell sarcoma & $\mathrm{t}(12 ; 22)(\mathrm{q} 13 ; \mathrm{q} 12)$ & EWSR1-ATF1 (EWS-ATF1) fusion \\
\hline Malignant rhabdoid tumor & Deletion of $22 q$ & HSNF5 (INI1) deletion or mutation \\
\hline
\end{tabular}

Adapted from Skubitz KM and D'Adamo [87] and from [14]. Gene symbols are those provided in the Human Genome Nomenclature Database (www.genenames.org). Previous names of genes are given in parentheses

then causes malignant transformation. There are alternative forms of the EWS/FLII gene that exist secondary to variations in the locations of the EWS and FLI1 genomic breakpoints. The most common form is type $1(60 \%)$, in which the first seven exons of EWS join to exons 6-9 of FLI1. Type 2 represents $25 \%$ of the various forms and includes FLI1 exon 5. Overall, type 1 fusion is associated with a significantly better prognosis, as it encodes a less active chimeric transcription factor.
Synovial sarcomas are soft tissue sarcomas in which approximately $30 \%$ occur in patients less than 20 years of age. These tumors tend to develop on the extremities and the most common site of metastasis is the lung. Synovial sarcomas harbor a unique chromosome translocation in which the SYT gene on chromosome 18 is translocated with the SSX gene on chromosome X. Pathologic subtypes of synovial sarcomas include mixed epithelial and spindle cell. Some of the poor prognostic factors include stage III/IV disease, 
truncal location, degree of necrosis and mitosis, and elevated age at diagnosed. Synovial sarcomas are typically more responsive to chemotherapy as compared to other sarcomas.

Desmoplastic small round cell tumor is a histological variant of a small round blue cell tumor and is distinguished by the presence of translocation $t(11 ; 22)(\mathrm{p} 13 ; \mathrm{q} 12)$, which has been characterized as a fusion of the WT1 and EWS genes. These tumors have a male predominance and most frequently involve the abdomen, pelvis, or tissues around the testes. Desmoplastic small round cell tumors invade locally but may spread to the lungs or elsewhere. The lesions are typically PET positive, which is useful for staging purposes. There is an overall poor prognosis, but these tumors tend to respond to multi-agent chemotherapy (with frequent relapses). The role of radiotherapy in the treatment of desmoblastic small round cell tumors is unclear at this time.

Epithelioid sarcoma is a type of NRSTS with uncertain histogenesis which displays multilineage differentiation and is secondary to inactivation of the SMARC/INII gene. Typical presentation is a slow growing firm nodule based in the deep soft tissue. Prognosis differs based on site of origin of the tumor, as the distal type, involving the hand, has a more benign course overall. The proximal type predominantly affects adults and involves the axial skeleton and proximal sites. This type is highly aggressive and has a propensity for lymph node metastases. One series of 30 pediatric patients with epithelioid sarcoma, with median age at presentation of 12 years, reported responses to chemotherapy in $40 \%$ of patients using sarcoma-based regimens. Sixty percent of these patients were alive 5 years after initial diagnosis.

MPNST are a type of NRSTS associated with neurofibromatosis 1 (NF1), but may also occur sporadically. In NF1 patients, $2-16 \%$ of nodular plexiform neurofibromas will transform into MPNSTs. Favorable features include localized disease, small tumor size, lower stage, and tumor location on an extremity as the primary site. Furthermore, there are variable reports noting that non-NF1 cases have a better prognosis. Overall, unresectable or metastatic disease has a poor prognosis while chemotherapy is associated with limited responses.

\section{Presentation, workup, and staging}

The majority of NRSTSs present as painless swelling; however, some may also present with signs and symptoms of neurologic abnormality from nerve compression, vascular compression, or bowel dysfunction when arising from the retroperitoneum. Swelling as the first symptom occurs in $75 \%$ of patients with NRSTS. While it is not surprising, the increasing tumor size, higher stage, and nodal spread are poor prognostic factors, the time interval from first symptom to diagnosis appears to be inversely correlated with survival for pediatric sarcomas [32]. Systemic symptoms such as fever, weight loss, or anorexia are rare. However, there is an association of hypoglycemic hypophosphatemic rickets with hemangiopericytoma and hyperglycemia with lung fibrosarcoma. One may speculate that such observations may one day be explained by juxtaposed deleted genes on a chromosomal segment encoding for a metabolic pathway and a tumor suppressor function. The typical workup starts with a plain radiograph of the affected area looking for evidence of soft tissue mass, calcification, and/or destruction of adjacent bone. Computed tomography (CT) and magnetic resonance imaging (MRI) are critical radiographic studies when evaluating the tumor extent, pattern of infiltration, and adjacent structures, as well as to allow for surgical and radiotherapeutic planning of treatment. To complete staging evaluation, chest radiograph, and thoracic CT scanning are commonly pursued in order to visualize the most common distant metastatic site, the lungs [33-38]. There has recently been an increased use of metabolic scanning techniques, including thallium and positron emission tomography (PET). PET scans determine glucose metabolism rate in the tumor and may be utilizing to correlate with tumor grade and to monitor therapeutic response $[39,40]$.

Staging pediatric NRSTS can be accomplished using the 2010 American Joint Committee on Cancer Staging (Table 2) [41], which incorporates the following: tumor size $(\mathrm{T} 1 \leq 5 \mathrm{~cm}$, $\mathrm{T} 2>5 \mathrm{~cm})$ and depth $(\mathrm{a}=$ superficial, $\mathrm{b}=$ deep $)$, nodal involvement $(\mathrm{N})$, distant metastases $(\mathrm{M})$, and histologic grade $(\mathrm{G})$. It is well-known that tumor size and resectability are important characteristics in predicting outcomes for pediatric NRSTS.

Table 2 The AJCC staging for soft tissue sarcoma

\begin{tabular}{lllll}
\hline \multirow{2}{*}{ Stage IA } & T1a & N0 & M0 & G1 \\
Stage IB & T1b & N0 & M0 & G1 \\
& T2a & N0 & M0 & G1 \\
\multirow{3}{*}{ Stage IIA } & T2b & N0 & M0 & G1 \\
& T1a & N0 & M0 & G2 \\
Stage IIB & T1b & N0 & M0 & G2, 3 \\
& T2a & N0 & M0 & G2 \\
& T2b & N0 & M0 & G2 \\
Stage III & T2b & N0 & M0 & G2 \\
& T2a, T2b & N0 & M0 & G3 \\
Stage IV & Any T & N1 & M0 & Any G \\
& Any T & Any N & M1 & Any G \\
\hline
\end{tabular}

$\mathrm{T} 1$ is defined as tumor less than or equal to $5 \mathrm{~cm}$ in greatest length with $\mathrm{T} 2$ greater than $5 \mathrm{~cm}$. The "a" designated a superficial tumor as located exclusively above the superficial fascia without invasion of the fascia; "b" designates a deep tumor is located either exclusively beneath the superficial fascia, superficial to the fascia with invasion of or through the fascia, or both superficial yet beneath the fascia. N1 and M1 are positive findings for nodal or distant metastatic spread. The $G$ designates a grade on a three-point scale of G1 well differentiation; G2 moderate differentiation, G3 poor or undifferentiation. Source: Edge et al. [88] 
But rather than TNM staging, risk stratification is more commonly employed for NRSTS. A retrospective analysis from St. Jude Children's Research Hospital in 2002 identified a statistically significant difference in 5-year survival probability in patients based on surgical risk stratification into three distinct groups: (1) grossly resected non-metastatic disease (89 \% 5-year survival); (2) initially unresectable, nonmetastatic disease (56\% 5-year survival), and (3) metastatic disease (15\% 5-year survival) [42-44]. Overall, adverse risk factors include metastatic disease, tumor size $>5 \mathrm{~cm}$, highgrade histology, positive surgical margins, intra-abdominal primary tumor site, and omission of postoperative radiotherapy in localized disease (see Fig. 3).

\section{Tumor grading}

Histologic grading has very important implications in predicting outcome of patients with NRSTS. Furthermore, it is crucial that clinicians understand the criteria and uncertainties associated with grading, as many treatment decisions depend on pathologic interpretation. There are primarily two grading systems for pediatric NRSTSs that we will focus on for this review. The first system, developed by the Pediatric Oncology Group (POG), labels tumors in the following way: grade 1, tumors with low tendency for malignancy; grade 2, tumors with fewer than five mitoses per ten high-powered fields or less than $15 \%$ geographic necrosis; and grade 3 , tumors known to be clinically aggressive by virtue of histologic diagnosis and with more than four mitoses per ten high-powered fields or more than $15 \%$ geographic necrosis (see Table 3) [45]. One review of this POG grading system found a $73 \%$ mortality in grade 3 lesions and $15 \%$ mortality in grade 1 and 2 tumors [46].

The second commonly used grading system, which is predominantly used worldwide, is the French Federation of Cancer Centres (FNCLCC) system, despite being largely based on adult cases [47]. This is a three-tiered grading system that has a point scoring system based on tumor histology, necrosis, and mitosis that has clinical appeal secondary to its reproducibility and simplicity (see Tables 4 and 5) [48]. The main criticism is that there are some histologic subtypes (e.g., pleomorphic undifferentiated sarcoma, alveolar soft parts sarcoma) that do not have characteristics which recapitulate normal tissues and cannot be easily scored in terms of differentiation.

Recently, Khoury et al. directly compared the POG and FNCLCC grading systems for pediatric NRSTSs [49]. In this study, 130 tumors were graded using both the POG and FNCLCC grading systems, and it was determined that both grading systems were equally effective in predicting eventfree survival. Interestingly, the POG system appeared to upgrade tumors in comparison to the FNCLCC system, and a conclusion was made that the FNCLCC system was superior to the POG system for tumors of intermediate grade. Furthermore, the mitotic index cutoff was noted to be a highly relevant grading parameter needing further study in a prospective trial.

There are certain types of pediatric NRSTS that have a low potential for metastasis, and surgery alone is the mainstay of therapy. These types include infantile fibrosarcoma, desmoid tumors (or aggressive fibromatosis), angiomatoid malignant fibrous histiocytoma, dermatofibrosarcoma, and hemangiopericytoma (in infants and young children). Infantile (congenital) fibrosarcoma is distinguished by its age of onset. Tumors which develop in children less than 5 years of age have a more benign behavior and are typically managed surgically. A fibrosarcoma that develops in older children is akin to adult-type soft tissue sarcoma. Desmoid tumors are locally infiltrating tumors with no metastatic risk. Surgical
Fig. 3 Risk stratification of NRSTS by primary tumor respectability and metastasis. From ref. [42]. Reprinted with permission. (C) 2002 American Society of Clinical Oncology. All rights reserved

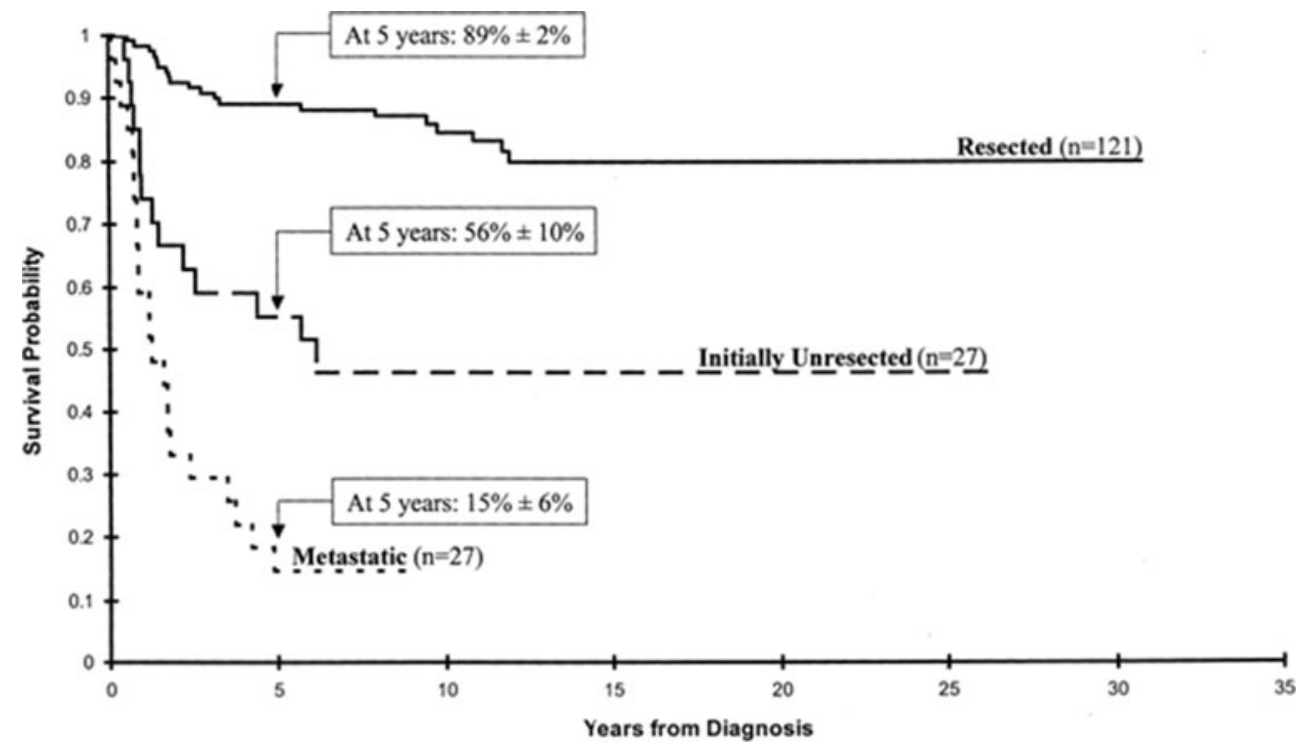


Table 3 POG grading system for pediatric NRSTS

$\begin{array}{cl}\text { Grade } & \text { Liposarcoma: myxoid and well differentiated } \\ 1 & \text { Deep-seated dermatofibrosarcoma protuberans } \\ & \text { Fibrosarcoma: well differentiated or infantile ( }<5 \text { year) } \\ & \text { Hemangiopericytoma: well differentiated } \\ & \text { or infantile }(<5 \text { year }) \\ & \text { Well-differentiated malignant peripheral nerve sheath tumor } \\ & \text { Angiomatoid malignant fibrous histiocytoma } \\ \text { Grade } & \text { All NRSTSs not in grades } 1 \text { or } 3 ;<15 \% \text { of tumor shows } \\ 2 & \begin{array}{l}\text { geographic necrosis, } \\ \text { or mitotic index is }<5 \text { mitoses } / 10 \text { high-power fields }\end{array} \\ \text { Grade } & \text { Fibrosarcoma with }>15 \% \text { of tumor with geographic necrosis } \\ 3 & \text { or mitotic index }>5 \text { mitoses } / 10 \text { high-power fields } \\ & \text { Liposarcoma: pleomorphic, round cell } \\ & \text { Mesenchymal chondrosarcoma } \\ & \text { Extraskeletal osteosarcoma } \\ & \begin{array}{l}\text { Malignant triton tumor } \\ \text { Alveolar soft part sarcoma }\end{array}\end{array}$

From ref. [3]

control can be difficult depending on location of the tumor, as the need to preserve normal structures is extremely important. There is a high potential for local recurrence after surgical resection alone but overall has a highly variable natural history, including the potential for spontaneous regression. Mutations in exon 3 of the beta-catenin gene are seen in over $80 \%$ of tumors. In treating these lesions with radiotherapy, gross versus microscopic tumor is controlled equally well using 5060 gray (Gy) [50]. Additionally, chemotherapy with sunitinib has been shown to have some efficacy [51], in addition to other chemotherapy regimens used in order to avoid the toxicity of high dose radiotherapy in a child.

\section{Treatment principles}

Aside from the aforementioned low-grade pediatric sarcomas that may be treated with surgery alone, the majority of NRSTS may be considered to have adult-like soft tissue sarcoma biologic characteristics. Of course exceptions exist, for example, embryonal sarcoma of the liver as a distinctive and rare pediatric cancer now often included under the NRSTS rubric. It is now differentiated from biliary rhabdomyosarcomas and has unique therapeutic challenges [52-54]. But in general, optimal therapeutic management of pediatric NRSTS requires a multi-disciplinary approach, with surgical resection, radiotherapy, and chemotherapy utilized as individual or combined therapy. Surgical resection is an important component of management for most soft tissue sarcomas. As in adults with extremity soft tissue sarcomas, less than $10 \%$ of patients undergo amputation. A wide, en bloc excision $(>1-2-\mathrm{cm}$ margins) is acceptable for low-grade, T1a lesions, while
Table 4 Tumor differentiation scores of sarcoma in the French Federation of Cancer Centres system of grading soft tissue sarcomas

\begin{tabular}{ll}
\hline Diagnosis & Differentiation \\
score
\end{tabular}

From Guillou et al. [89]

MPNT malignant peripheral nerve tumor, PNET primitive neuroectodermal tumor, $M F H$ malignant fibrous histiocytoma

conservative surgery plus radiotherapy is the standard for all other STSs with an overall local control of $85-90 \%$. For patients presenting with localized, low-grade tumors, complete surgical resection alone (if able to obtain margins that are tumor-free) or re-excision if original margins were positive is typically utilized. In cases where tumor margins are positive and further resection is not feasible, postoperative radiotherapy or brachytherapy should be considered. The COG 
Table 5 French Federation of Cancer Centres system of grading soft tissue sarcomas

\begin{tabular}{ll}
\hline Parameter & Criterion \\
\hline $\begin{array}{l}\text { Tumor differentiation } \\
\text { Score }=1\end{array}$ & $\begin{array}{c}\text { Sarcoma histologically very similar } \\
\text { to normal adult mesenchymal tissue } \\
\text { Sarcoma for defined histological } \\
\text { subtype (e.g., myxoid MFH) }\end{array}$ \\
Score $=2$ & $\begin{array}{c}\text { Sarcoma of uncertain type, embryonal } \\
\text { and undifferentiated sarcomas }\end{array}$ \\
Mitosis count & \\
Score $=1$ & $0-9 / 10$ HPF \\
Score $=2$ & $10-19 / 10$ HPF \\
Score $=3$ & $\geq 20 / 10$ HPF \\
Microscopic tumor necrosis & \\
Score $=0$ & No necrosis \\
Score $=1$ & $\leq 50 \%$ tumor necrosis \\
Score $=2$ & $>50 \%$ tumor necrosis \\
Histological grade & \\
Grade 1 & Total score 2 or 3 \\
Grade 2 & Total score 4 or 5 \\
Grade 3 & Total score 6,7, or 8 \\
\hline
\end{tabular}

Modified from Guillou et al. [89] $M F H$ malignant fibrous histiocytoma, $H P F$ high-power field

ARST0032 trial for low-grade NRSTS divides patients into two separate clinical groups. Clinical group I underwent definitive surgical resection followed by no radiotherapy and clinical group II undergoes definitive surgical resection followed by radiotherapy to a total dose of $55.8 \mathrm{~Gy}$ if it is deemed that a local recurrence would result in significant morbidity. A note is made that this is a lower radiation dose than in adult practice in which retrospective reviews show that postoperative radiation doses in the 60-66-Gy range is optimal [55].

Management of localized, high-grade NRSTS again utilizes complete surgical resection alone for small tumors, if all margins are negative after initial resection or additional re-excision in the setting of an initial marginal resection with positive margins. This differs in stark contrast to adult STS practice in which adjuvant radiotherapy is felt to be beneficial for improved local control. We await follow-up data from the recently closed COG ARST0032 trial to see if this subset of patients has an excessive local recurrence rate. But, similar to low-grade tumors, postoperative radiotherapy or brachytherapy is recommended if tumor margins are positive and further resection is not possible. In the setting of complete excision with negative tumor margins, the size of the tumor influences the use of adjuvant therapy. Furthermore, the use of preoperative radiotherapy in NRSTS has been infrequent.

More specifically in COG ARST0032, adjuvant therapy after definitive surgical resection was determined by tumor size.
Those patients with tumors greater than $5 \mathrm{~cm}$ went on to receive postoperative radiotherapy to a total dose of $55.8 \mathrm{~Gy}$ as well as five cycles of ifosfamide and doxorubicin chemotherapy. Those patients with tumors less than $5 \mathrm{~cm}$ were divided based upon whether a fascial plane was disrupted during the surgical resection. Patients with an unbroken fascial plane did not receive radiotherapy, while those with a broken fascial plane receiving adjuvant radiotherapy to a total dose of $55.8 \mathrm{~Gy}$.

The actual evidence for postoperative radiotherapy after surgical resection in NRSTS is sparse but has been nicely reviewed by Million and Donaldson [56]. The POG protocol 8653 intended to study the role of adjuvant radiotherapy in children with surgically resectable NRSTS [10]. Local therapy was standardized to consist of surgery alone for patients ( $n=55$ patients) with complete resection with surgical margin defined as a "cuff of normal tissue." Those with marginal resections or positive margins ( $n=25$ patients) were to receive radiotherapy using doses that were age adjusted: under age 6 received 35 Gy with an additional 10 Gy boost, while older patients received 45 Gy with a 5-Gy boost. Protocol guidelines were improperly followed, but results indicate higher local control rates with radiotherapy for marginal excisions in high-grade tumors [57]. Specifically, of those patients with high-grade tumors, there were six out of 26 local failures with surgery alone compared to one out of 13 local recurrences for surgery and radiotherapy. Moreover, the POG grade 3 patients had a $52 \%$ survival at 5 years compared to the POG grade 1-2 patients with a $92 \% 5$-year survival. In addition, four retrospective reviews of the St. Jude Children's Research Hospital experience with NRST considered the role of adjuvant radiotherapy [3, 42-44, 58]. In totally, resected disease (clinical group I), postoperative radiotherapy appeared to reduce local recurrence only in high-grade disease. Furthermore, in patients with positive surgical margins (clinical group II), radiotherapy reduced local recurrences $(p<0.001)$. These findings are of critical importance because there will be many clinical situations in which radical surgery is not possible or is possible and refused secondary to unacceptable functional, cosmetic, or psychological consequences. In these patients, radiotherapy may play a crucial role by allowing more conservative surgery with similar rates of local control.

Investigators from the University of Florida have reported on 95 NRSTS patients, including those who were young adults who had resectable disease that was managed with either preoperative radiotherapy (RT) to a median dose of 50.4 Gy or postoperative RT to a median dose of $61 \mathrm{~Gy}$ [59]. Overall local control was $12 \%$ at 5 years. Patients with close or positive margins defined as less than $1 \mathrm{~cm}$ had local recurrences in eight out of 30 patients $(27 \%$ ) compared to four out of 64 patients $(6 \%)$ with negative margins. There were no associations based on grade, but as this was not a randomized trial, low-grade tumors tended to not be irradiated in 
comparison to high-grade tumors. Finally, all local recurrences lead to ultimate death of the patient.

Treatment of advanced disease presents a unique clinical challenge. For those patients with locally advance tumors, multimodality therapy is utilized. The role of chemotherapy is beyond the scope of this review. Adjuvant chemotherapy in pediatric NRSTS was originally defended on the basis of the significant risk of metastatic disease and local recurrence in high-grade lesions, retrospective comparisons, and the success of chemotherapy in rhabdomyosarcoma. The most active single agents are doxorubicin and ifosfamide [60]. Other active agents are dacarbazine, actinomycin $\mathrm{D}$, vincristine, etoposide, and cyclophosphamide $[3,12,33,35,36$, 61-63]. But, increasing certain histologies of NRSTS are being recognized as being more chemosensitive. Combinations of doxorubicin and ifosfamide or gemcitabine and Taxol are increasing being utilized. Integration with concurrent radiotherapy is problematic for certain drugs such as doxorubicin, which may markedly increase acute toxicities such as skin reactions. But, increasingly, the role of preoperative chemo- and radiotherapy is being explored. Metastatic disease tends to be treated with chemotherapy in the adult population with more reliance on surgical resection in NRSTS. In these cases, chemotherapy and radiation therapy are utilized for residual or high-grade disease.

For those patients presenting with oligometastatic pulmonary disease, surgical resection of metastases should be considered, and the use of stereotactic radiosurgery needs further investigation. COG ARST0032 approached metastatic disease based on initial tumor grade. For high-grade tumors, patients were then divided into two groups, grossly resected versus unresected, where they received adjuvant chemotherapy plus radiotherapy versus neoadjuvant chemoradiotherapy, respectively. For low-grade tumors, if all disease was resected, independent of microscopic margin status, these patients went on to observation alone. If all disease was not resected, they entered in the high-grade arm of the algorithm where further therapy was assigned.

Ferrari et al. (2011) published a retrospective pooled analysis from the US and Europe investigating prognostic variables and treatment modalities in 304 patients with initially unresectable but nonmetastatic NRSTS (akin to rhabdomyosarcoma clinical group III) [64]. This study noted that there was similar negative prognostic implication of particular tumor characteristics compared to adult soft tissue sarcoma, with those poor prognostic features including large tumor size, MPNST histology, older patient, and axial location of tumor. When examining response rate to chemotherapy, a $41 \%$ response rate was recorded (in terms of complete response and major partial response). This was $57 \%$ if minor responses were included. In addition, it was reported that final outcome was directly correlated with response to initial chemotherapy. Particular importance of local therapy was also emphasized in this study, as local progression and relapse was the major cause of treatment failure and patients able to undergo complete delayed resection had the best outcome. From a radiotherapy perspective, the use of radiotherapy also was directly correlated with a better survival on multivariate analysis. It was noted that radiotherapy improved survival after incomplete resection, but had little or no benefit after complete surgery. However, the authors do describe a selection bias for those patients who received radiotherapy, as these patients were a selected subset with unfavorable features. Furthermore, the use of radiotherapy was not homogeneous and was highly variable over time between centers included in the study. Radiotherapy was the only local modality in 72 patients. An additional 72 patients underwent surgery and radiotherapy with the vast majority undergoing radiotherapy postoperatively. The median total radiation dose was 54 Gy for patients receiving RT alone or postoperative RT for positive margins. Patients who had a complete surgical resection and who received RT had a median radiation dose of $50 \mathrm{~Gy}$. Overall, this study concluded that aggressive multimodal treatment strategies should be pursued with effort to get a patient to a complete surgical resection. This study has also pointed out that there are histology-specific predictors of chemotherapy response seen in other studies, which in turn impact on prognosis. Specifically, synovial cell sarcoma is a relatively chemosensitive tumor while malignant peripheral nerve sheath tumor tended to be more chemoresistant. Investigators at COG in designing future NRSTS studies are contemplating using preoperative chemoradiation for locally advanced tumors in which lower radiation doses will be justified if there is a good response to chemotherapy or in the case of chemosensitive histologies.

Another University of Florida study focused on unresectable, nonmetastatic NRSTS patients that documented relatively poor results associated with radiotherapy and chemotherapy [65]. Of the 19 patients in this small study, the median radiation dose was $55.2 \mathrm{~Gy}, 12$ patients received chemotherapy, and 13 patients had high-grade tumors. The 5 -year local control rate was only $40 \%$. Nine of the 13 recurrences were local only, but all recurrences ultimately resulted in patient death, half of whom never developed metastatic disease. This study highlighted the need for more intensive therapies aimed at improving local control for unresectable NRSTS.

\section{Radiotherapy}

Radiotherapy for pediatric NRSTS may be delivered after biopsy, but before definitive surgery (preoperatively), postoperatively, intraoperatively, or as definitive therapy. The arguments for preoperative over postoperative irradiation 
include the following. First, preoperative treatment will produce partial regression of the tumor, and the resection may be less extensive than if the surgery had been done first. Second, preoperative treatment may decrease the risk of autotransplantation of the tumor in the surgical bed and may also decrease the risk of intravascular seeding. Third, in preoperative treatment, the clinically and radiographically demonstrable areas of risk are treated while other tissues are protected $[66,67]$. In addition, because postoperative treatment must cover all surgically manipulated areas, the volume receiving radiotherapy is quite often much larger. Finally, the postoperative surgical bed may be poorly vascularized, so the concern regarding tumor cell hypoxia in this setting has led to the requirement of higher radiation doses. Results of the Canadian randomized trial in adult STS (discussed further below) suggest an equivalence of 50 Gy preoperatively to 66-70 Gy postoperatively [68].

When determining proper radiotherapy treatment margins, it is important to remember that margins do not cross uninvolved bone or fascia that is not breached by tumor. Margins may be relatively tight for low-grade lesions. Historically, radiation field treatment margins have been quite large $(5-10 \mathrm{~cm})$ for adult, high-grade extremity STS, but may be able to be decreased. MRI imaging may play a critical role in establishing of proper tumor margins for adult STS and for NRSTS by extrapolation. White and colleagues performed a histologic assessment of peritumoral edema in soft tissue sarcoma [69]. In this study, they sought to evaluate whether satellite tumor cells could be identified in the tissues surrounding a soft tissue sarcoma and whether their presence correlated with T2 signal on MRI. Fifteen patients with high-grade extremity and truncal soft tissue sarcoma underwent preoperative MRI and 12 of these patients received gadolinium contrast. The extent of the T2 signal was then determined, and the presence of tumor cells in the surrounding tissues of pathologic specimens was correlated with the MRI findings. None of the patients underwent preoperative chemotherapy or radiotherapy. The maximal tumor size for patients in this study was $13.8 \mathrm{~cm}$ (mean value). The extent of peritumoral T2 signal was $2.5 \mathrm{~cm}$ and the extent of peritumoral contrast enhancement was $1.1 \mathrm{~cm}$. Of note, the maximal length of T2 signal was usually in the superior-inferior direction. It was noted that tumor cells were present beyond the tumor margin in ten of the 15 cases. In six of these cases, tumor cells were $<1 \mathrm{~cm}$ from the tumor margin while, in four cases, tumor cells were located 1-4 $\mathrm{cm}$ from the tumor margin. There were 88 anatomic regions available for histologic evaluation. For 24 regions with negative peritumoral edema, 21 had no presence of tumor cells beyond tumor margins, while three did. For 64 regions with positive peritumoral edema, $53 \mathrm{had}$ no presence of tumor cells beyond tumor margins, while 11 did. Overall, areas of edema were twice as likely to contain tumor cells and only three of the 16 anatomic regions with tumor cells were outside of the regions with edema (histologically or by MRI). Furthermore, there was no correlation between tumor size and presence of tumor cells beyond tumor margin.

Krasin and colleagues have reported on a small series of 32 high-grade NRSTS cases in which 2-cm margins around gross tumor volumes were chosen [70]. Median doses for preoperative and postoperative radiotherapy were 45 and 60 Gy, respectively. The 3-year local recurrence rate was $4 \%$. Local recurrences were in the high dose radiation volume and correlated with those patients who had marginal resections.

As previously mentioned, one of the primary goals in the treatment of NRSTS is to maximize tumor control, while preserving function. Therefore, careful consideration of the benefits and complications secondary to preoperative versus postoperative radiotherapy should be carefully considered. Preoperative radiotherapy requires smaller field sizes and lower doses, but is also associated with higher rates of wound complications. However, long-term complications may be better with smaller doses and smaller fields. Pisters et al. (2007) describe the "trade-off" between preoperative versus postoperative radiotherapy highlighting the particular issues associated with these two modalities [71].

Furthermore, O'Sullivan and colleagues performed a prospective randomized trial in which they aimed to determine whether the timing of external beam radiotherapy affected the frequency of wound healing complications in soft tissue sarcoma of the limbs [68]. This randomized trial by the NCI Canada included 190 patients enrolled between 1994 and1997. Eligible patients included those who had limb soft tissue sarcoma without the presence of metastasis. Patients were stratified according to tumor size, $\leq 10 \mathrm{~cm}$ or $>10 \mathrm{~cm}$, and then randomized to receive preoperative radiotherapy consisting of 50 Gy in 25 fractions with a 16-20-Gy postoperative boost in the setting of positive surgical margins (94 patients) versus postoperative radiotherapy consisting of 66 Gy in 33 fractions (96 patients). Surgery and radiotherapy were separated by 3-6 weeks in both groups. Primary endpoint was frequency of wound complications within 120 days of surgery and secondary endpoints included local control, development of distant metastases, progression-free survival, and overall survival. The trial had to be closed prematurely before reaching its accrual goal of 266 patients secondary to an interim analysis which showed significantly different outcomes. The median follow-up was 3.3 years. Fourteen patients in the preoperative radiotherapy group had positive surgical margins and ten underwent postoperative radiotherapy boost. The median field size was $333 \mathrm{~cm}^{2}$ in the preoperative group and $416 \mathrm{~cm}^{2}$ in the postoperative group ( $p=0.01$ ). Overall, $35 \%$ of the patients in the preoperative group suffered wound complications compared to 
$17 \%$ in the postoperative group. In addition, there was no difference between groups in rates of local recurrence, regional/distant recurrence, or progression-free survival. Overall survival was slightly higher in the preoperative versus postoperative group ( $p=0.0481)$, but the study was not powered to look at survival.

Davis et al. (2005) examined late radiation morbidity in patients randomized to preoperative versus postoperative radiotherapy for extremity soft tissue sarcoma [72]. This NCIC study enrolled 129 patients, and morbidity was evaluated at a single time-point: 2 years. Functional status was assessed using the Musculoskeletal Tumor Society Salvage Score (MSTS) in which the clinician rated pain, strength, joint stability, range of motion, and global function, as well as the Toronto Extremity Salvage Score (TESS) in which the patient evaluated difficulties in performing ADLs, self-care, and mobility. Furthermore, fibrosis and joint stiffness were evaluated using the EORTC/RTOG Late Radiation Toxicity Criteria and edema was evaluated using Stern's Rating Scale for Edema. Results indicated that patients treated with postoperative radiotherapy tended to have greater subcutaneous fibrosis ( $48 \%$ vs. $32 \%, p=0.07$ ) with no statistically significant different between groups for amount of joint stiffness or edema. Furthermore, there was no statistically significant difference in function between the two groups for either the MSTS or TESS scores ( $p=0.08$ and 0.17 , respectively). Of note, radiotherapy field size was predictive or greater rates of fibrosis $(p=0.002)$, joint stiffness $(p=0.006)$, and marginally predictive of edema $(p=0.06)$. Also, the 6.9year median follow-up data were presented at the American Society for Clinical Oncology 2004 Annual Meeting and grade $2-4$ toxicity was noted to be $68 \%$ in the preoperative group versus $86 \%$ in the postoperative group ( $p=0.002)$.

A common conundrum occurs when patients undergoing preoperative radiotherapy are found to have close or positive surgical margins when they ultimately undergo surgery. While such microscopic tumor residua in the surgical bed may still be destined to die from the prior effects of radiotherapy, postoperative radiotherapy has historically been a consideration. Recent data from the Canadian investigators, however, suggest that there is little benefit to this practice of delivering a postoperative boost [73].

Brachytherapy may play an important role in limbsparing surgery for NRSTS [74]. Brachytherapy is often, but not always, combined with pre- or postoperative external beam treatment. Potential advantages of brachytherapy include: its radiobiologic effectiveness, which is increased by the delivery of a high dose of radiotherapy over a few days rather than several weeks; concentrated dose given deep within the tumor bed; increased sparing of the surrounding normal tissue and overlying skin secondary to the rapid reduction of dosage; and the convenience of shorter treatment time [75-77]. A more detailed exploration of the use of brachytherapy in low-grade tumors was pursued at MSKCC because their original randomized trial did not include a large number of patients with low-grade NRSTS. From 1982 to 1992,45 patients with low-grade tumors were randomized to brachytherapy versus observation. There was no benefit to brachytherapy in terms of local recurrence (approximately $76 \%$ local control in each arm, $p=0.60$ ) or overall survival $(p=0.38)$ [78].

Furthermore, in two additional studies, patients with high-grade NRSTS with negative margins had a local control rate of $89 \%$ and $94 \%$, respectively, when treated with brachytherapy, compared to $59 \%$ versus $77 \%$ in those with positive margins. In patients with high-grade tumors and positive margins treated with brachytherapy and external beam radiotherapy, the local control rate was $90 \%$ [78-80]. From the investigators' point of view, these two randomized trials argue in favor of adjuvant brachytherapy for resected high-grade tumors, surgery alone in the treatment of resected low-grade tumors $<5 \mathrm{~cm}$, and for consideration of postoperative external beam radiotherapy for resected low-grade tumors $>5 \mathrm{~cm}$ because of the remaining high risk of local recurrence in these tumors (i.e., 20-25\%). Early in the MSKCC trials, there was a significantly higher rate of wound complications in patients treated with brachytherapy compared to observation (44\% vs. $14 \%$, $p=0.0006)$. The higher rate of complications has been improved by a guiding principle of not loading patients with radioactivity until the fifth postoperative day [81].

Newer external beam modalities offer the potential for better sparing of normal tissues and hence an improvement in the therapeutic ratio. The use of intensity modulated radiotherapy to better conform the high dose radiation distribution to the tumor region may well reduce developmental toxicities, but comes with the theoretical problem of increased integral dose which may increase the later risk for secondary cancers $[82,83]$. Image guidance techniques improve the accuracy of radiotherapy delivery and may prove useful to further reduce radiotherapy treatment margins. Particle therapy, such as protons, is enthusiastically under investigation and has shown preliminary benefit in Ewing's sarcoma and rhabdomyosarcomas [84-86].

\section{Conclusions}

Overall, the treatment approach to pediatric NRSTS should have a multimodality focus. When considering the use of radiotherapy, careful thought of many patient characteristics and tumor factors must be reviewed in order to provide the best treatment plan. Certain lowgrade, small tumors are well managed with surgery alone. But, as we have described in this review, preoperative radiotherapy, although it has a propensity for greater 
frequency of perioperative wound complications over postoperative radiotherapy (larger volumes, higher doses), can lead to less long-term morbidity. Furthermore, we emphasized the importance of using tighter radiotherapeutic treatment margins including regions of peritumoral edema identified on MRI to aid in the design of treatment fields. More consideration for preoperative external beam radiotherapy in the treatment of their NRSTS needs to be considered, especially for those patients with high-risk features, where possible. And the future use of newer radiation modalities such as proton radiotherapy and selected use of brachytherapy may help to reduce toxicities.

Conflict of interest The authors declare that they have no conflict of interest.

\section{References}

1. Conrad EU 3rd, Bradford L, Chansky HA (1996) Pediatric softtissue sarcomas. Orthop Clin North Am 27(3):655-664

2. Greenberg J (1982) Epithelioid sarcoma. Med Pediatr Oncol 10 (5):497-500

3. Pappo AS et al (1999) Soft tissue sarcomas in children. Semin Surg Oncol 16(2):121-143

4. Ries LAG, SEER Program (National Cancer Institute (U.S.) (1999) Cancer incidence and survival among children and adolescents: United States SEER program 1975-1995. In: Lynn A, Gloecker R et al (eds) SEER pediatric monograph 1999. National Cancer Institute, Bethesda, p 182

5. Weihkopf $\mathrm{T}$ et al (2008) Incidence and time trends of soft tissue sarcomas in German children 1985-2004-a report from the population-based German Childhood Cancer Registry. Eur J Cancer 44(3):432-440

6. Ferrari A et al (2011) Soft tissue sarcoma across the age spectrum: a population-based study from the Surveillance Epidemiology and End Results database. Pediatr Blood Cancer 57(6):943-949

7. Jemal A et al (2008) Cancer statistics, 2008. CA Cancer J Clin 58 (2):71-96

8. Jemal A et al (2009) Cancer statistics, 2009. CA Cancer J Clin 59 (4):225-249

9. Pratt CB et al (1998) Treatment of unresectable or metastatic pediatric soft tissue sarcomas with surgery, irradiation, and chemotherapy: a Pediatric Oncology Group study. Med Pediatr Oncol 30(4):201-209

10. Pratt CB et al (1999) Role of adjuvant chemotherapy in the treatment of surgically resected pediatric nonrhabdomyosarcomatous soft tissue sarcomas: a Pediatric Oncology Group Study. J Clin Oncol 17(4):1219

11. Fletcher CDM Pathology and genetics of tumours of soft tissue and bone [electronic resource]: WHO classification of tumours, volume 5 2002. World Health Organization, Geneva, p 415

12. Horowitz ME et al (1986) Therapy for childhood soft-tissue sarcomas other than rhabdomyosarcoma: a review of 62 cases treated at a single institution. J Clin Oncol 4(4):559-564

13. Demetri GD et al (2002) Efficacy and safety of imatinib mesylate in advanced gastrointestinal stromal tumors. N Engl J Med 347(7):472480
14. Spunt SL, Skapek SX, Coffin CM (2008) Pediatric nonrhabdomyosarcoma soft tissue sarcomas. Oncologist 13(6):668-678

15. Aman $P$ et al (1992) Rearrangement of the transcription factor gene CHOP in myxoid liposarcomas with $\mathrm{t}(12 ; 16)(\mathrm{q} 13 ; \mathrm{p} 11)$. Genes Chromosom Cancer 5(4):278-285

16. Bennicelli JL, Barr FG (2002) Chromosomal translocations and sarcomas. Curr Opin Oncol 14(4):412-419

17. Fletcher CD et al (1996) Correlation between clinicopathological features and karyotype in lipomatous tumors. A report of 178 cases from the Chromosomes and Morphology (CHAMP) Collaborative Study Group. Am J Pathol 148(2):623-630

18. Fletcher JA et al (1991) Diagnostic relevance of clonal cytogenetic aberrations in malignant soft-tissue tumors. N Engl J Med 324 (7):436-442

19. Jeon IS et al (1995) A variant Ewing's sarcoma translocation (7;22) fuses the EWS gene to the ETS gene ETV1. Oncogene 10 (6):1229-1234

20. Ladanyi M, Bridge JA (2000) Contribution of molecular genetic data to the classification of sarcomas. Hum Pathol 31(5):532-538

21. Lee YF et al (2003) Molecular classification of synovial sarcomas, leiomyosarcomas and malignant fibrous histiocytomas by gene expression profiling. Br J Cancer 88(4):510-515

22. Mitelman F (2000) Recurrent chromosome aberrations in cancer. Mutat Res 462(2-3):247-253

23. Mugneret $\mathrm{F}$ et al (1988) Chromosomes in Ewing's sarcoma. II. Nonrandom additional changes, trisomy 8 and $\operatorname{der}(16) \mathrm{t}(1 ; 16)$. Cancer Genet Cytogenet 32(2):239-245

24. Pedeutour F et al (1999) Structure of the supernumerary ring and giant rod chromosomes in adipose tissue tumors. Genes Chromosom Cancer 24(1):30-41

25. Pedeutour F et al (1994) Complex composition and coamplification of SAS and MDM2 in ring and giant rod marker chromosomes in well-differentiated liposarcoma. Genes Chromosom Cancer 10(2):85-94

26. Quade BJ et al (2004) Molecular pathogenesis of uterine smooth muscle tumors from transcriptional profiling. Genes Chromosom Cancer 40(2):97-108

27. Ren B et al (2003) Gene expression analysis of human soft tissue leiomyosarcomas. Hum Pathol 34(6):549-558

28. Sreekantaiah C et al (1994) Chromosomal aberrations in soft tissue tumors. Relevance to diagnosis, classification, and molecular mechanisms. Am J Pathol 144(6):1121-1134

29. Tomescu O, Barr FG (2001) Chromosomal translocations in sarcomas: prospects for therapy. Trends Mol Med 7(12):554-559

30. Turc-Carel C et al (1986) Cytogenetic studies of adipose tissue tumors. I. A benign lipoma with reciprocal translocation $\mathrm{t}$ $(3 ; 12)(\mathrm{q} 28 ; \mathrm{q} 14)$. Cancer Genet Cytogenet 23(4):283-289

31. van de Rijn M, Fletcher JA (2006) Genetics of soft tissue tumors. Annu Rev Pathol 1:435-466

32. Ferrari A et al (2010) The symptom interval in children and adolescents with soft tissue sarcomas. Cancer 116(1):177-183

33. Raney B et al (1987) Treatment of children with neurogenic sarcoma. Experience at the Children's Hospital of Philadelphia, 1958-1984. Cancer 59(1):1-5

34. Raney RB Jr et al (1979) Results of multimodal therapy for children with neurogenic sarcoma. Med Pediatr Oncol 7(3):229-236

35. Raney RB Jr (1979) Proceedings of the Tumor Board of the Children's Hospital of Philadelphia: alveolar soft-part sarcoma. Med Pediatr Oncol 6(4):367-370

36. Raney RB Jr (1991) Chemotherapy for children with aggressive fibromatosis and Langerhans' cell histiocytosis. Clin Orthop Relat Res 262:58-63

37. Salloum E et al (1990) Diagnostic and therapeutic problems of soft tissue tumors other than rhabdomyosarcoma in infants under 1 year of age: a clinicopathological study of 34 cases treated at the Institut Gustave-Roussy. Med Pediatr Oncol 18(1):37-43 
38. Suit HD et al (1985) Preoperative, intraoperative, and postoperative radiation in the treatment of primary soft tissue sarcoma. Cancer 55(11):2659-2667

39. Jones DN et al (1996) Monitoring of neoadjuvant therapy response of soft-tissue and musculoskeletal sarcoma using fluorine-18-FDG PET. J Nucl Med 37(9):1438-1444

40. McCarville MB et al (2005) PET/CT in the evaluation of childhood sarcomas. AJR Am J Roentgenol 184(4):1293-1304

41. Edge SB, American Joint Committee on Cancer (2010) AJCC cancer staging manual, 7th edn. Springer, New York, p 648

42. Spunt SL et al (2002) Clinical features and outcome of initially unresected nonmetastatic pediatric nonrhabdomyosarcoma soft tissue sarcoma. J Clin Oncol 20(15):3225-3235

43. Pappo AS et al (1999) Metastatic nonrhabdomyosarcomatous softtissue sarcomas in children and adolescents: the St. Jude Children's Research Hospital experience. Med Pediatr Oncol 33(2):76-82

44. Spunt SL et al (1999) Prognostic factors for children and adolescents with surgically resected nonrhabdomyosarcoma soft tissue sarcoma: an analysis of 121 patients treated at St. Jude Children's Research Hospital. J Clin Oncol 17(12):3697-3705

45. Parham DM et al (1995) Nonrhabdomyosarcomatous soft tissue sarcomas of childhood: formulation of a simplified system for grading. Mod Pathol 8(7):705-710

46. Rao BN (1993) Nonrhabdomyosarcoma in children: prognostic factors influencing survival. Semin Surg Oncol 9(6):524-531

47. Deyrup AT, Weiss SW (2006) Grading of soft tissue sarcomas: the challenge of providing precise information in an imprecise world. Histopathology 48(1):42-50

48. Trojani M et al (1984) Soft-tissue sarcomas of adults; study of pathological prognostic variables and definition of a histopathological grading system. Int J Cancer 33(1):37-42

49. Khoury JD et al (2010) Grading of nonrhabdomyosarcoma soft tissue sarcoma in children and adolescents: a comparison of parameters used for the Federation Nationale des Centers de Lutte Contre le Cancer and Pediatric Oncology Group Systems. Cancer 116(9):2266-2274

50. Ballo MT, Zagars GK, Pollack A (1998) Radiation therapy in the management of desmoid tumors. Int J Radiat Oncol Biol Phys 42 (5):1007-1014

51. George S et al (2009) Multicenter phase II trial of sunitinib in the treatment of nongastrointestinal stromal tumor sarcomas. J Clin Oncol 27(19):3154-3160

52. Baron PW et al (2007) Undifferentiated embryonal sarcoma of the liver successfully treated with chemotherapy and liver resection. J Gastrointest Surg 11(1):73-75

53. Urban CE et al (1993) Undifferentiated (embryonal) sarcoma of the liver in childhood. Successful combined-modality therapy in four patients. Cancer 72(8):2511-2516

54. Steiner M et al (1989) Undifferentiated (embryonal) sarcoma of the liver. A clinicopathologic study of a survivor treated with combined technique therapy. Cancer 64(6):1318-1322

55. Mundt AJ et al (1995) Conservative surgery and adjuvant radiation therapy in the management of adult soft tissue sarcoma of the extremities: clinical and radiobiological results. Int J Radiat Oncol Biol Phys 32(4):977-985

56. Million L, Donaldson SS (2012) Resectable pediatric nonrhabdomyosarcoma soft tissue sarcoma: which patients benefit from adjuvant radiation therapy and how much? ISRN Oncol 2012:341408

57. Marcus RB Jr (1996) Current controversies in pediatric radiation oncology. Orthop Clin North Am 27(3):551-557

58. Blakely ML et al (1999) The impact of margin of resection on outcome in pediatric nonrhabdomyosarcoma soft tissue sarcoma. J Pediatr Surg 34(5):672-675

59. Smith KB et al (2011) Adjuvant radiotherapy for pediatric and young adult nonrhabdomyosarcoma soft-tissue sarcoma. Int $\mathrm{J}$ Radiat Oncol Biol Phys 81(1):150-157
60. Ferrari A et al (2005) The role of adjuvant chemotherapy in children and adolescents with surgically resected, high-risk adulttype soft tissue sarcomas. Pediatr Blood Cancer 45(2):128-134

61. Wenger J, Davidson R (1984) Fibrosarcoma of the leg. Med Pediatr Oncol 12(3):209-211

62. Mazanet R, Antman KH (1991) Adjuvant therapy for sarcomas. Semin Oncol 18(6):603-612

63. Oosterom AT, Unnik JAM, EORTC Soft Tissue and Bone Sarcoma Group (1986) Management of soft tissue and bone sarcomas. Monograph series of the European Organization for Research on Treatment of Cancer. Raven Press, New York, p 309

64. Ferrari A et al (2011) Non-metastatic unresected paediatric nonrhabdomyosarcoma soft tissue sarcomas: results of a pooled analysis from United States and European groups. Eur J Cancer 47(5):724-731

65. Smith KB et al (2011) Definitive radiotherapy for unresectable pediatric and young adult nonrhabdomyosarcoma soft tissue sarcoma. Pediatr Blood Cancer 57(2):247-251

66. Cheng EY et al (1996) Soft tissue sarcomas: preoperative versus postoperative radiotherapy. J Surg Oncol 61(2):90-99

67. Tanabe KK et al (1994) Influence of surgical margins on outcome in patients with preoperatively irradiated extremity soft tissue sarcomas. Cancer 73(6):1652-1659

68. O'Sullivan B et al (2002) Preoperative versus postoperative radiotherapy in soft-tissue sarcoma of the limbs: a randomised trial. Lancet 359(9325):2235-2241

69. White LM et al (2005) Histologic assessment of peritumoral edema in soft tissue sarcoma. Int J Radiat Oncol Biol Phys 61(5):1439-1445

70. Krasin MJ et al (2010) Preliminary results from a prospective study using limited margin radiotherapy in pediatric and young adult patients with high-grade nonrhabdomyosarcoma soft-tissue sarcoma. Int J Radiat Oncol Biol Phys 76(3):874-878

71. Pisters PW, O’Sullivan B, Maki RG (2007) Evidence-based recommendations for local therapy for soft tissue sarcomas. J Clin Oncol 25(8):1003-1008

72. Davis AM et al (2005) Late radiation morbidity following randomization to preoperative versus postoperative radiotherapy in extremity soft tissue sarcoma. Radiother Oncol 75(1):48-53

73. Al Yami A et al (2010) Positive surgical margins in soft tissue sarcoma treated with preoperative radiation: is a postoperative boost necessary? Int J Radiat Oncol Biol Phys 77(4):1191-1197

74. Merchant TE et al (2000) Brachytherapy for pediatric soft-tissue sarcoma. Int J Radiat Oncol Biol Phys 46(2):427-432

75. Schray MF et al (1990) Soft tissue sarcoma. Integration of brachytherapy, resection, and external irradiation. Cancer 66(3):451-456

76. Gerbaulet A et al (1985) Iridium afterloading curietherapy in the treatment of pediatric malignancies. The Institut Gustave Roussy experience. Cancer 56(6):1274-1279

77. Shiu MH et al (1991) Brachytherapy and function-saving resection of soft tissue sarcoma arising in the limb. Int J Radiat Oncol Biol Phys 21(6): 1485-1492

78. Pisters PW et al (1996) Long-term results of a prospective randomized trial of adjuvant brachytherapy in soft tissue sarcoma. J Clin Oncol 14(3):859-868

79. Alekhteyar KM et al (1996) The effect of combined external beam radiotherapy and brachytherapy on local control and wound complications in patients with high-grade soft tissue sarcomas of the extremity with positive microscopic margin. Int J Radiat Oncol Biol Phys 36(2):321-324

80. Harrison LB et al (1993) Long-term results of a prospective randomized trial of adjuvant brachytherapy in the management of completely resected soft tissue sarcomas of the extremity and superficial trunk. Int J Radiat Oncol Biol Phys 27(2):259-265

81. Devlin PM, Harrison LB (1997) Brachytherapy for soft tissue sarcomas. Cancer Treat Res 91:107-128

82. Lin C et al (2012) Effect of radiotherapy techniques (IMRT vs. 3D-CRT) on outcome in patients with intermediate-risk 
rhabdomyosarcoma enrolled in COG D9803-a report from the Children's Oncology Group. Int J Radiat Oncol Biol Phys 82(5): $1764-1770$

83. Sterzing F et al (2009) Intensity modulated radiotherapy (IMRT) in the treatment of children and adolescents - a single institution's experience and a review of the literature. Radiat Oncol 4:37

84. Childs SK et al (2012) Proton radiotherapy for parameningeal rhabdomyosarcoma: clinical outcomes and late effects. Int J Radiat Oncol Biol Phys 82(2):635-642

85. Rombi B et al (2012) Proton radiotherapy for pediatric Ewing's sarcoma: initial clinical outcomes. Int J Radiat Oncol Biol Phys 82 (3):1142-1148
86. Yock $\mathrm{T}$ et al (2005) Proton radiotherapy for orbital rhabdomyosarcoma: clinical outcome and a dosimetric comparison with photons. Int J Radiat Oncol Biol Phys 63(4):1161-1168

87. Skubitz KM, D'Adamo DR (2007) Sarcoma. Mayo Clinic Proceedings 82(11):1409-1432

88. Edge SB, Byrd DR, Compton CC et al (2009) American Joint Committee on Cancer (AJCC) cancer staging manual, 7th edn. Springer, Berlin

89. Guillou et al (1997) Comparative study of the National Cancer Institute and French Federation of Cancer Centers Sarcoma Group grading systems in a population of 410 adult patients with soft tissue sarcoma. J Clin Oncol 15:350-362 